respondent was asked to indicate 'True', 'False' or 'I don't know'. Correct answers were contrasted with incorrect and 'I don't know' answers to give a general overview of STI knowledge in this population.

Results Preliminary analysis of the first 159 questionnaires returned showed variable knowledge of STI transmission, treatment and possible consequences. Students had a good knowledge of transmission, with $88 \%$ knowing that STIs can be spread even when symptoms are not present. However, knowledge about treatment and potential sequelae was poor, with $58 \%$ of respondents indicating they did not know whether Gonorrhoea could usually be treated with antibiotics, and $44 \%$ indicating that they were not aware that some kinds of cancer could be caused by STIs.

Conclusion This analysis indicates that some areas of STI knowledge could be improved in this population. This is important because knowledge can be addressed through public health interventions.

Disclosure of interest statement The authors declare no conflicts of interest.

\section{P03.12 RECRUITING TO SEXUAL HEALTH STUDIES: POSSIBLE STRATEGIES IDENTIFIED THROUGH FOCUS GROUP DISCUSSIONS}

${ }^{1} \mathrm{HJ}$ Denison*, ${ }^{1,2}$ EM Dennison, ${ }^{1} \mathrm{~A}$ Jutel. ${ }^{1}$ Victoria University of Wellington, Wellington, New Zealand; ${ }^{2}$ Medical Research Council Lifecourse Epidemiology Unit, University of Southampton, Southampton, UK

\subsection{6/sextrans-2015-052270.240}

Background Recruiting individuals to take part in studies about sexual health can often be difficult due to the sensitive nature of the study topic. As a result, the sample may be less representative of the general population than desired. Understanding potential participants' views, motivations and concerns may aid in the design of a recruitment protocol that maximises participation and minimises bias.

Methods During the initial stages of a study concerning sexually transmitted infections (STIs) at a New Zealand university health centre, a series of focus groups were carried out in order to understand acceptable methods of recruitment. The focus group participants were sought from the study target population and were recruited via posters displayed around the university. Three focus groups of between five and seven participants were conducted. Discussions were audio-recorded and transcribed verbatim. Content analysis methodology was employed using a constant comparative method.

Results A key theme that emerged centred around the timing of any approach; students almost unanimously agreed that an approach prior to an STI consultation would be more acceptable than after. Reasons given included wanting to "just get out of there" after seeing the clinician, as well as having something to do during idle time in the waiting room. Many students suggested that they would be less likely to take part after receiving a STI test result that was positive, as they would "not want to think about it".

Conclusion Understanding students' views around STI testing and research participation helped us to develop a protocol that was sympathetic to the target population's attitudes and desires, thus improving its acceptability and attractiveness to this population. This information may be useful to other researchers planning sexual health surveys in similar populations.
Disclosure of interest statement The authors declare no conflicts of interest.

\section{P03.13 THE PROJECT CONNECT PARENTAL MONITORING INTERVENTION: POPULATION-LEVEL EFFECTS ON ADOLESCENT PERCEPTIONS OF PARENTAL ENFORCEMENT OF FAMILY RULES}

PJ Dittus*, CR Harper, E Hoo, KA Ethier. Centers for Disease Control and Prevention

\subsection{6/sextrans-2015-052270.241}

Background The relationship between parental monitoring and adolescent risk behaviour is well established. Both overall parental knowledge of adolescent activities and parental enforcement of family rules are associated with a decreased likelihood of sexual initiation. Few interventions exist to improve parents' monitoring efforts with their children, particularly at a populationlevel. Project Connect is a low-cost highly sustainable approach using distribution of print modules to influence parental monitoring practices.

Methods Twelve high schools and fourteen middle schools in a public school district in Los Angeles, California were matched on geography, teen birth and Chlamydia rates and then randomly assigned to intervention and comparison conditions. Survey data were collected from 28,470 male and female students sampled cross-sectionally every year for five years, from randomly selected required classes. The sample was $78.6 \%$ Latino and 10.6\% African American; the mean age was 15.4. Intervention materials were mailed to all parents in intervention schools (more than 33,000). Analyses were conducted in SPSS 21 with linear mixed models that accounted for students being potentially sampled in multiple years.

Results Across all years, intervention school students reported higher enforcement of family rules than comparison school students $\left(\mathrm{F}_{[1,28470]}=5.25, p=0.022\right)$. Although student reports of family rules enforcement increased across the five years for both intervention and comparison students $\left(\mathrm{F}_{[4,28470]}=9.66, p\right.$ $<00.001)$ there was a significant interaction between condition and time, with intervention students' reports of family rules increasing more than comparison students' $\left(\mathrm{F}_{[4,28470]}=2.54, p\right.$ $=0.038)$. Intervention effects on student reported parental knowledge were non-significant.

Conclusions We observed a population-level intervention effect on adolescent perceptions of parental enforcement of family rules using a brief, easily administered print-based intervention. Project Connect materials may be used to increase this protective factor against sexual initiation in a variety of contexts where parents of adolescents may be reached, including schools, physician's offices and clinics, and community-based organisations.

Disclosure of interest statement Nothing to declare.

\section{P03.14 HIGH PREVALENCE OF LACTOBACILLIS CRISPATUS AMONG ADOLESCENT GIRLS ATTENDING SECONDARY SCHOOL IN TANZANIA}

${ }^{1} \mathrm{SC}$ Francis*, ${ }^{2} \mathrm{~V}$ Jespers, ${ }^{1,3} \mathrm{C}$ Hansen, ${ }^{3} \mathrm{~J}$ Irani, ${ }^{1} \mathrm{~K}$ Baisley, ${ }^{1,3} \mathrm{~A}$ Andreasen, ${ }^{4} \mathrm{~S}$ Nnko, ${ }^{2} \mathrm{~T}$ Crucitti, ${ }^{4} \mathrm{C}$ Changalucha, ${ }^{1} \mathrm{RJ}$ Hayes, ${ }^{1,3} \mathrm{D}$ Watson-Jones, ${ }^{2} \mathrm{~A}$ Buve. ${ }^{1}$ London School of Hygiene and Tropical Medicine; ${ }^{2}$ Institute of Tropical Medicine, Antwerp; ${ }^{3}$ Mwanza Intervention Trials Unit/National Institute for Medical Research; ${ }^{4}$ National Institute for Medical Research, Mwanza, Tanzania

10.1136/sextrans-2015-052270.242 